TITLE:

\title{
An Architecture of a Network Controller for QoS Management in Home Networks with Lots of IoTDevices and Services
}

$\operatorname{AUTHOR}(S)$ :

Kotani, Daisuke

CITATION:

Kotani, Daisuke. An Architecture of a Network Controller for QoS Management in Home Networks with Lots of IoTDevices and Services. 2019 16th IEEE Annual Consumer Communications \& Networking Conference (CCNC) 2019: 18493018.

ISSUE DATE:

2019-1

URL:

http://hdl.handle.net/2433/252431

RIGHT:

(c) 2019 IEEE. Personal use of this material is permitted. Permission from IEEE must be obtained for all other uses, in any current or future media, including reprinting/republishing this material for advertising or promotional purposes, creating new collective works, for resale or redistribution to servers or lists, or reuse of any copyrighted component of this work in other works.; This is not the published version. Please cite only the published version.; この論文は出版社版 でありません。引用の際には出版社版をご確認ご利用ください。 


\title{
An Architecture of a Network Controller for QoS Management in Home Networks with Lots of IoT Devices and Services
}

\author{
Daisuke Kotani \\ Academic Center for Computing and Media Studies \\ Kyoto University \\ Kyoto, Japan \\ kotani@media.kyoto-u.ac.jp
}

\begin{abstract}
More and more IoT services are introduced in home, and they will consume many network resources in home networks including an uplink to the Internet, but the resources are sometimes insufficient to host all services. Traditionally, QoS control is applied to handle such situation by prioritizing important traffic. However, in IoT at home, it is hard to find important traffic because it depends on the context that is greatly different among homes, such as services and the life of residents. In addition, administrators in home networks can rarely understand the context and configure the whole system including IoT devices and services. This paper proposes an architecture of the network controller that automatically estimates and prioritizes important traffic under such situation. The controller under the proposed architecture provides three interfaces to ask each party, IoT devices, service providers, and users, for input about its information. Then, the controller automatically estimates the important traffic based on the input and applies the estimated policy to the network in a centralized way. This paper also shows key points of designing each interface according to the information that each party knows.
\end{abstract}

Index Terms-IoT, Controller, QoS, QoE, Home Network

\section{INTRODUCTION}

There are great expectations of IoT (Internet of Things) enabled services to enhance people's life in various fields including smart city, manufacturing, transportation, and many others. Smart home [1] is one of fields that are attracted as an application of IoT. The applications of the smart home range from monitoring and visualization of data captured by sensors embedded into home such as energy management [2] and human behavior monitoring for safety [3], to control home appliances for improving human life [4]. As the population of elderly grows, robots have also much attention for healthcare and elderly support at home [5].

Nowadays IoT devices at home sometimes need to communicate with cloud services to achieve their roles. Examples include robots that work with functions provided as cloud services so called cloud robotics [6], wearable devices to monitor health of each person [7], activity recognition using data sensed by many devices [8].

As a result, more data will be delivered between home and cloud services over the Internet when more IoT devices and services are introduced at home. However, quality of an Internet connection from home is sometimes not enough to support many services simultaneously. Although average download throughput is reported to be tens of Mbps [9], which may be enough for most of services used at home, throughput to the Internet and within the network sometimes becomes low due to temporal congestion, bad wireless conditions, etc.

To keep quality of life of residents at home with many IoT devices and services under such insufficient network resources, it will be important to manage traffic in home networks so that services that have huge impact on the life of residents are less affected by such insufficient resources. Traditionally, QoS mechanisms are introduced to prioritize important traffic to overcome such situation, like IntServ [10], DiffServ [11], traffic engineering, and Software Defined Networking [12]. We may be able to use these technologies for home networks.

One problem to apply QoS mechanisms to home networks with many IoT devices and services is to identify important traffic for maintaining quality of life of residents at home. It is readily understood that important traffic depends on the context, like residents at home and their activities. When no one is at home, traffic to deliver pictures taken by surveillance cameras to the cloud will have high priority because of home security, and traffic from/to robots that interact with the residents has less priority than those from the cameras because no one uses the robots. On the other hand, when a resident directly interacts with the robots, such as talking with the robots, traffic from the robots usually has higher priority than those from the cameras, because response delay to the resident has negative impact on the quality of life of the resident.

In addition, no one has enough knowledge to identify important traffic for residents at home and configure networking devices because of diversity of IoT devices and services and lack of skilled administrators at each home. IoT devices and device manufactures know the details of the devices, e.g., the amount of traffic and intervals of sending data, but do not know the importance at services using data. IoT service providers know the importance of data exchanged with devices, but they usually have no knowledge about the importance of their services on the life of residents. The residents may know the importance of services for their life, but the details of services 
and devices are usually hidden from the users.

This paper proposes an architecture of a controller that manages a home network with many IoT services in a centralized way, for increasing quality of life of residents at home. We assume that the network can be dynamically configured in a centralized way, e.g. by SDN using OpenFlow and the controller behaves as an SDN controller. The controller provides three interfaces to accept input from each party involved, one for IoT devices, another for service provides, and the other for residents at home. The controller automatically estimates the global priority of each flow from the input, and configures networking devices like wireless access points, switches and CPE (Customer Premises Equipment) according to the global priority and network status.

The contributions of this paper are summarized as follows:

- We have proposed an architecture of the controller of a home network with many IoT devices and services without administrator who knows the whole system and the context, which will be a common limitation in many home networks

- We have provided key points of designing interfaces for IoT devices, service providers, and residents at home, with consideration of data that they can provide.

\section{RELATED WORK}

There are several standards for IoT devices, services and interoperable platforms. FIWARE [13] and W3C WoT (Web of Things) [14] are examples of such platform. FIWARE is originally designed for a platform of smart city to collect data from sensors in the city and distribute data to many applications. W3C WoT aims for a more general IoT platform to make IoT devices and services interoperable. Both FIWARE and W3C WoT has standard specifications to describe the capability of devices, such as data types that each device can sense and provide as well as metadata of each device. Our proposed architecture makes use of the same model as much as possible so that IoT device manufacturers do not have to learn various models to describe device capabilities.

Some platforms focused on specific use cases of IoT, such as smart home [1], [15].They aim to support complex tasks like message exchange between devices, interaction with cloud services and device discovery. If network resources are insufficient to host these platforms, support at the network layer will be needed to smoothly operate these platforms, preferably by coordinating these platforms and networks. For example, Jang, et al. presented the case of bandwidth management [16].

In IoT, QoS can be regarded broader than those in traditional networks [17]. Metrics of QoS in IoT include accuracy of data and timeliness of data delivery as well as latency and jitter. Based on this insight, many algorithms have been proposed to implement QoS for IoT environment [17]-[19]. Our focus is how to use these QoS mechanisms rather than improvement of such mechanisms and their implementation. There will be many types of IoT services, and requirements to transmit data vary in each service. So, to make use of these QoS mechanisms effectively, there is a need to control a network with enough knowledge of IoT devices and services.

One thing we need to consider from the network viewpoint is that some IoT devices are tightly coupled with cloud services. Some robots, an example of IoT devices that are expected to be everywhere in the near future, communicate with cloud services to implement their features, so called Cloud Robotics [6]. Rospeex [20] is an example of cloud robotics platforms for human-robot spoken dialogue. Rospeex provides an API to retrieve results of speech recognition and output audio of text, and it uses cloud services for speech recognition and synthesis. We need to consider such characteristics, that is, when controlling IoT devices, the devices autonomously communicate with cloud services.

\section{Proposed ARchitecture}

\section{A. Target Environment and Assumption}

Figure 1 shows environment where we assume the proposed architecture is applied.

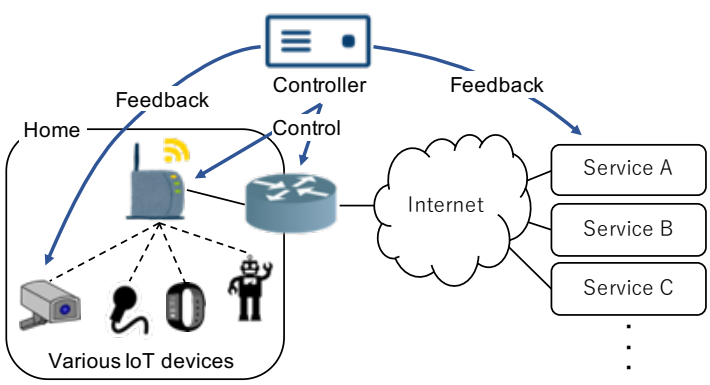

Fig. 1. Assumption of environment and use case of the proposed architecture.

There are many IoT devices at home, and one or more cloud services exchange data with these devices over the Internet. The proposed architecture is implemented as a controller that configures QoS parameters on networking devices in home networks. The controller optionally gives feedback to devices and services so that the services can optimize their quality under the network status reported by the controller.

In home networks, we assume that administrators can rarely know and optimize systems as a whole, because three parties are involved in IoT services on home networks as follows and they hide their details from others.

IoT devices (device manufactures) provide IoT devices to the users. Some IoT devices implement a part of their features as cloud services provided by the manufacturers or thirdparty, like cloud robotics. Data and functions on the devices are accessed from the service providers that the users have selected. Examples include microphones, cameras, wearable sensors, environmental sensors, robots, and home appliances.

Service Providers offer services to the users using data and functions on IoT devices owned by the users. Services retrieve data from one or more devices, process the data, and control the devices. Example services include home monitoring, remote control of home appliances, health monitoring 
and emergency calls/dialogues with robots. Importance of data differs among services. For example, a home monitoring service may always process pictures taken by cameras to find a suspicious person, but if network resources are insufficient to transmit the pictures, the service may use sound recorded by microphones instead of the pictures. A health monitoring service may use data sensed on several wearable devices at different frequency depending on health status of the users.

Users are residents at home. They own IoT devices at their home and use services offered by service providers. Importance of services depends on status of the users and their home. For example, a home monitoring service is less important when one or more adult residents are at home because they can perceive abnormal status by themselves. Instead, services that directly interact with them may be more important to improve their life.

\section{B. Architecture Overview}

Figure 2 shows an overview of the proposed architecture of the controller.

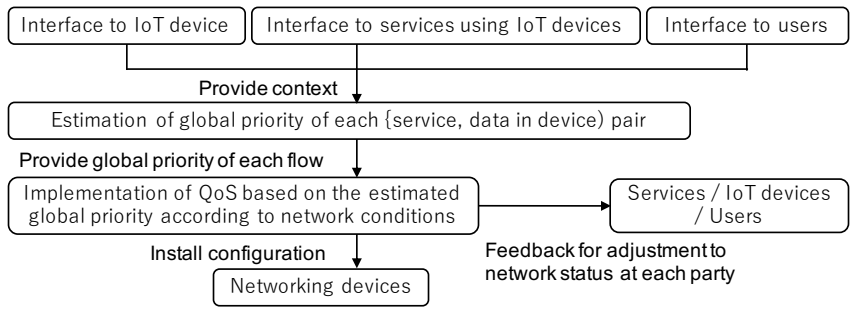

Fig. 2. An overview of the proposed architecture.

1) Interfaces to IoT Devices / Services / Users: The first step is that the controller obtains context information related to IoT devices, services and users. To receive input, the controller has three interfaces that are suitable for each party. Each interface is designed according to the following principle.

Interface to IoT Devices is to learn what kinds of data a device can send and receive. This aim is similar to device abstractions in existing IoT platforms such as FIWARE [13] and WoT [14], which is expressed as a set of (property/attribute (property hereafter), type of its value) pairs. We use the same model with them so that device definitions on FIWARE or WoT can be reused as much as possible. Such information does not depend on the services and the users, so the devices are assumed to automatically provide their information via this interface.

In addition, for each property, the controller needs to know cloud services where the devices communicate to provide and process data (Coflow [21] in the context of IoT, so we call IoT Coflow hereafter), because the objective of applying QoS cannot be achieved without consideration of such IoT Coflow.

Interface to Services is to learn which data (property) in the devices a service accesses. The service registers a set of properties on devices that the users have, along with the endpoint of the service (IP address, URL, etc.) that communicates with the devices.
As described in Section III-A, the importance of data may depend on services, that is, some data may be less important than others to provide a service. The services are also asked to provide such importance as a priority of each property.

Interface to Users is to learn which services are important for the users' life. The users are expected to provide a set of (service, priority for their life) pairs via this interface.

2) Estimation of Global Priority of Each (Service, Property on a Device) Pair: The second step is to estimate the global priority of each (service, property on a device) pair for users' life using input from the interfaces. The global priority is assigned from the viewpoint of integrating all IoT devices and services used by the residents at home.

Basically, this estimation gives more preference on services that the users think important, and on (service, property on a device) pair that are important to provide services to the users.

There is a tradeoff between two strategy of the estimation. One is to maximize the number of services that the users can be offered at the same time. To achieve this, (service, property on a device) pairs that have high priorities provided by the services should have high global priorities. The other is to maximize the quality of each service. This may be achieved by assigning high priorities to (service, property on a device) pairs of the important services.

3) Implementation of QoS Based on Global Priority: The third step is to apply the global priority to network traffic considering network status. We assume we can reuse existing sophisticated algorithms for QoS shown in Section II, as well as simple greedy algorithms that packs the traffic into the network in the highest priority order for bandwidth management. The controller configures networking devices to apply QoS to each traffic.

Another role of this step is to feedback the results of this step to the devices and the services. Although this feedback and the use of this feedback at the devices and the services are optional, we expect them to use this feedback to improve the quality of each service at the device or the service level by changing ways of using data. For example, a home monitoring service stops using pictures taken by cameras and uses audio recorded by microphones because of the feedback of insufficient network resources and trials to continue to monitor the home. When the services and the devices change to decide ways to process data, they provide new information through the corresponding interface at the controller, and the controller updates network configuration with new global priorities.

\section{PRototype Implementation}

We have developed the first prototype controller based on the proposed architecture with limited capabilities that make an admission control of flows at one OpenFlow switch. The interfaces are designed as REST API, and the estimation and implementation components have their own REST API so that we can easily replace these components to better ones. The estimation algorithm is designed to maximize the number of services that the residents can use, because unavailability of services may greatly decrease the quality of life. 


\section{EXAmPle Use CASE}

Suppose that residents use two services, a dialogue robot service with a companion robot and a home monitoring service. The dialogue robot service enables users to make a conversation with the robot. The service retrieves speech recognition results from the robot, understands the context, and creates and sends text to the robot for the speech synthesis by the robot. The robot accesses cloud services over the Internet for the speech recognition and synthesis. The service may retrieve pictures taken by a camera on the robot for emotion recognition to understand the context precisely. This service is defined in our system as follows:

Device - Robot: Speech recognition result, text for speech synthesis, and picture taken by the camera are defined as individual properties. IoT Coflows to cloud services are defined for speech recognition result and text for speech synthesis.

Service: The service registers the speech recognition result and the text for speech synthesis on the robot as the top priority, and the picture as the second priority. This is because making a speech is very important and the picture is an option.

The home monitoring service provides detection of suspicious activities when no one is at home. The service periodically retrieves pictures taken by cameras around the home, and notifies the users when a suspicious activity is detected. This service is defined in our system as follows:

Device - Camera: Each camera is registered separately, and has a property "picture."

Service: The service registers the pictures taken by the cameras. If there is an overlap of coverage between the cameras, the service may prioritize some cameras that cover the whole area with a small number of cameras.

The users prioritize the dialogue robot service over the home monitoring service when they are at home because they can monitor by themselves. The flows from the robot to the cloud services for speech recognition and synthesis as well as text transmission between the robot and the dialogue robot service are prioritized because such flows are specified as IoT Coflow. When they go out, they reverse the priorities of the services because no one uses the dialogue robot service, and the priorities of the coflows are decreased as well. In this way, our system tries to improve the user experience.

\section{CONCLUding REMARKS}

This paper proposes an architecture of the controller that manages QoS in home networks with many IoT devices and services so that users can get a good experience by using many services. One of important features is that the proposed architecture is designed under assumption that administrators rarely have good knowledge of the whole network and systems, which will be realistic in many homes. The automatic estimation of global priority by using input from three parties, IoT devices, services, and users, will contribute to derive the necessary policy for QoS in the home networks.

Future work includes extensive evaluation of interfaces from the perspective of real service development in realistic scenarios, designing more sophisticated algorithms for global priority estimation, integration of sophisticated QoS implementation algorithms, and interfaces of more IoT oriented protocols such as CoAP and MQTT.

\section{ACKNOWLEDGMENT}

The author would like to thank Hitoshi Morioka of SRC Software, Satoru Momiki, and members of ACCRA project for their valuable comments and assistance. This work is partly supported by the Commissioned Research of National Institute of Information and Communications Technology.

\section{REFERENCES}

[1] M. Soliman, T. Abiodun, T. Hamouda, J. Zhou, and C.-H. Lung, "Smart Home: Integrating Internet of Things with Web Services and Cloud Computing," in IEEE CloudCom 2013, Dec 2013, pp. 317-320.

[2] D.-M. Han and J.-H. Lim, "Smart home energy management system using IEEE 802.15. 4 and zigbee," IEEE Trans. Consum. Electron., vol. 56 , no. 3, pp. 1403-1410, 2010.

[3] N. Noury, et al., "Monitoring behavior in home using a smart fall sensor and position sensors," in 1st Annual International IEEE-EMBS Special Topic Conference on Microtechnologies in Medicine and Biology, 2000, pp. 607-610.

[4] J. Choi, D. Shin, and D. Shin, "Research and implementation of the context-aware middleware for controlling home appliances," IEEE Trans. Consum. Electron., vol. 51, no. 1, pp. 301-306, Feb 2005.

[5] K. Yamazaki, et al., "Home-Assistant Robot for an Aging Society," Proc. IEEE, vol. 100, no. 8, pp. 2429-2441, Aug 2012.

[6] J. Kuffner, "Cloud-Enabled Robotics," in IEEE-RAS Humanoids 2010 , Dec 2010.

[7] M. Hassanalieragh, et al., "Health Monitoring and Management Using Internet-of-Things (IoT) Sensing with Cloud-Based Processing: Opportunities and Challenges," in IEEE SCC 2015, June 2015, pp. 285-292.

[8] F. Cicirelli, et al., "On the Design of Smart Homes: A Framework for Activity Recognition in Home Environment," Journal of Medical Systems, vol. 40, no. 9, p. 200, Jul 2016.

[9] M. Isla, "The World's Internet Speeds Increased More than 30\% in 2017. Are You Keeping Up?" http://www.speedtest.net/insights/blog/globalspeed-2017/, accessed: 2018-07-31.

[10] R. Braden, D. Clark, and S. Shenker, "Integrated Services in the Internet Architecture: an Overview," RFC1633, IETF, June 1994.

[11] S. Blake, et al., "An Architecture for Differentiated Services," RFC2475, IETF, Dec 1998.

[12] M. F. Bari, S. R. Chowdhury, R. Ahmed, and R. Boutaba, "PolicyCop: An Autonomic QoS Policy Enforcement Framework for Software Defined Networks," in IEEE SDN4FNS 2013, Nov 2013, pp. 1-7.

[13] "FIWARE," https://www.fiware.org/.

[14] W. W. W. Consortium, "Web of Things at W3C," https://www.w3.org/WoT/.

[15] S. Amilcare, Francesco, R. Floriano, De, F. Domenico, and B. Domenico, "SmartHome: a domotic framework based on smart sensing and actuator network to reduce energy wastes," Proc.SPIE 9013, Wireless Sensing, Localization, and Processing IX, 910308, May, 2014.

[16] H. Jang and J. Lin, "SDN based QoS aware bandwidth management framework of ISP for smart homes," in IEEE SmartWorld/SCALCOM/UIC/ATC/CBDCom/IOP/SCI 2017, Aug 2017, pp. 16.

[17] L. Li, S. Li, and S. Zhao, "QoS-Aware Scheduling of Services-Oriented Internet of Things," IEEE Trans. Ind. Informat., vol. 10, no. 2, pp. 14971505, May 2014

[18] T. Tachibana, T. Furuichi, and H. Mineno, "Implementing and Evaluating Priority Control Mechanism for Heterogeneous Remote Monitoring IoT System," in Adjunct Proc. MOBIQUITOUS 2016, ACM, 2016, pp. 239-244.

[19] S. Aslam, N. U. Hasan, A. Shahid, J. W. Jang, and K.-G. Lee, "Device Centric Throughput and QoS Optimization for IoTsin a Smart Building Using CRN-Techniques," Sensors, vol. 16, no. 10, 2016.

[20] K. Sugiura and K. Zettsu, "Rospeex: A Cloud Robotics Platform for Human-Robot Spoken Dialogues," in IEEE/RSJ IROS 2015, Sept 2015 , pp. 6155-6160.

[21] M. Chowdhury and I. Stoica, "Coflow: A Networking Abstraction for Cluster Applications," in ACM HotNets-XI. ACM, 2012, pp. 31-36. 